PROCEEDINGS OF THE

AMERICAN MATHEMATICAL SOCIETY

Volume 128, Number 1, Pages 135-139

S 0002-9939(99)04980-1

Article electronically published on June 30, 1999

\title{
ON THE EXTREMALITY OF QUASICONFORMAL MAPPINGS AND QUASICONFORMAL DEFORMATIONS
}

\author{
SHEN YU-LIANG
}

(Communicated by Albert Baernstein II)

\begin{abstract}
Given a family of quasiconformal deformations $F(w, t)$ such that $\bar{\partial} F$ has a uniform bound $M$, the solution $f(z, t)(f(z, 0)=z)$ of the Löwnertype differential equation

$$
\frac{d w}{d t}=F(w, t)
$$

is an $e^{2 M t}$-quasiconformal mapping. An open question is to determine, for each fixed $t>0$, whether the extremality of $f(z, t)$ is equivalent to that of $F(w, t)$. The note gives this a negative approach in both directions.
\end{abstract}

\section{§1. INTRODUCTION}

This paper deals with the quasiconformal solutions $w=f(z, t)(f(z, 0)=0)$ of the following Löwner-type differential equation

$$
\frac{d w}{d t}=F(w, t)
$$

in the unit disk. The maximal dilatation $K[f]$ of $f$ can be estimated in terms of the sup norm of $\bar{\partial} F$. It is of interest to find out whether minimizing the sup norm of $\bar{\partial} F$ is equivalent to minimizing the maximal dilatation $K[f]$. To make this precise, we will need some definitions and notation.

In the terminology of Ahlfors [1], a complex-valued function $F(w)$ defined in a Jordan domain $\Omega$ is called a quasiconformal deformation if $F(w)$ is continuous in $\Omega \cup \partial \Omega$ and has locally $L^{2}$-generalized derivatives, $\partial F, \bar{\partial} F$, with $\bar{\partial} F \in L^{\infty}(\Omega)$. The deformation provides a way of generating a family of quasiconformal mappings, and is suitable for obtaining some distortion theorems (see Reich [6]).

Now for a homemorphism $h$ of the unit circle $\partial \Delta$ onto itself, denote by $Q C(h)$ the class of all quasiconformal mappings of the unit disk $\Delta=\{z:|z|<1\}$ with boundary values $h$. Then $Q C(h)$ is non-empty if and only if $h$ is quasisymmetric in the sense of Beurling-Ahlfors [2]. A quasisymmetric function $h$ then determines the extremal maximal dilatation $K_{h}$, defined as

$$
K_{h}=\inf \{K[f]: f \in Q C(h)\} .
$$

$f_{0} \in Q C(h)$ is extremal if $K\left[f_{0}\right]=K_{h}$.

Received by the editors December 23, 1997 and, in revised form, March 10, 1998.

1991 Mathematics Subject Classification. Primary 30C70, 30C62.

Key words and phrases. Quasiconformal mapping, quasiconformal deformation, extremality. Project supported by the National Natural Science Foundation of China.

(C)1999 American Mathematical Society 
Similarly, for a continuous function $H$ defined on $\partial \Delta$, denote by $Q D(H)$ the class of all quasiconformal deformations $F$ on $\Delta$ with boundary values $H$. GardinerSullivan [3] and Reich-Chen [7] respectively proved that $Q D(H)$ is non-empty if and only if $H$ is a Zygmund function, providing that $F$ satisfies the following normalized conditions:

$$
\operatorname{Re}[\bar{w} F(w)]=0 \quad(|w|=1), \quad F(0)=F(1)=0 .
$$

Recall that a continuous function $\phi(x)$ is called a Zygmund [9] function if it satisfies

$$
|\phi(x+t)-2 \phi(x)+\phi(x-t)|=O(t)
$$

for all real numbers $x$ and $t>0$. Define

$$
B_{H}=\inf \left\{\|\bar{\partial} F\|_{\infty}: F \in Q D(H)\right\}
$$

for a Zygmund function $H$. Then $F_{0} \in Q D(H)$ is extremal if $\left\|\bar{\partial} F_{0}\right\|_{\infty}=B_{H}$.

Now we can formulate our problem as follows. Given a family of quasiconformal deformations $F(w, t)$ being continuous on $\bar{\Delta} \times\{t \geq 0\}$ such that $\bar{\partial} F$ is uniformly bounded, that is, there exists some constant $M>0$ such that $|\bar{\partial} F(w, t)| \leq M$ for a.e. $w \in \Delta$ and all $t \geq 0$, it is known (see [6]) that the differential equation (1) has a unique solution $f(z, t)$ with the initial condition $f(z, 0)=z$, which is an $e^{2 M t}$-quasiconformal mapping on the unit disk $\Delta$. If, additionally, $F(w, t)$ satisfy the normalized conditions (2), then $f(z, t)$ map $\Delta$ onto itself with $f(0, t)=f(1, t)-$ $1=0$. An open question is to determine, for each fixed $t>0$, whether the extremality of $f(z, t)$ is equivalent to that of $F(w, t)$. After giving some preliminary results, we will give a negative approach to the question in both directions. On the other hand, we will give a sufficient condition under which the answer to the question is affirmative.

\section{§2. Preliminaries}

2.1. Let $A$ denote the Banach space of all functions $\phi$ holomorphic in $\Delta$ with the usual $L^{1}$-norm. The natural pairing:

$$
(\mu, \phi)=\iint_{\Delta} \mu \phi, \quad \mu \in L^{\infty}, \phi \in A,
$$

induces a linear map $P$ from $L^{\infty}$ onto $A^{*}$, the dual of $A$, defined by $P \mu(\phi)=(\mu, \phi)$. $\mu \in L^{\infty}$ is said to satisfy the Hamilton-Krushkal ([4], [5]) condition if $\|P \mu\|=\|\mu\|_{\infty}$.

We will use the following standard result.

Proposition 1 ([4], [5], [7], [8]). (1) $f$ is an extremal quasiconformal mapping iff its complex dilatation $\mu$ satisfies the Hamilton-Krushkal condition.

(2) $F$ is an extremal quasiconformal deformation iff its $\bar{\partial}$-derivative $\bar{\partial} F$ satisfies the Hamilton-Krushkal condition.

Note that $\mu \in L^{\infty}$ satisfies the Hamilton-Krushkal condition iff there exists a sequence $\left(\phi_{n}\right)$ in $A$ with $\left\|\phi_{n}\right\|=1$ such that $\iint_{\Delta} \mu \phi_{n} \rightarrow\|\mu\|_{\infty}$ as $n \rightarrow \infty$. Such a sequence $\left(\phi_{n}\right)$ is called a Hamilton sequence for $\mu$. It is said to be degenerating if $\phi_{n} \rightarrow 0$ locally uniformly in $\Delta$.

We will also need the following

Proposition $2([8])$. $\mu$ satisfies the Hamilton-Krushkal condition iff $\mu /\left(1-|\mu|^{2}\right)$ does. 
2.2. Let $f(z, t)$ be the solution of the system (1). As done in Reich [6], differentiating both sides of the equation

$$
\frac{d f(z, t)}{d t}=F(f(z, t), t)
$$

partially with respect to $z$ and $\bar{z}$ yields the relation

$$
f_{z}(z, t)^{2} \partial_{t} \mu(z, t)=\left(\left|f_{z}(z, t)\right|^{2}-\left|f_{\bar{z}}(z, t)\right|^{2}\right) \bar{\partial} F(f(z, t), t),
$$

or equivalently,

$$
\bar{\partial} F(f(z, t), t)=\frac{\partial_{t} \mu(z, t)}{1-|\mu(z, t)|^{2}} \cdot \frac{f_{z}(z, t)}{\overline{f_{z}(z, t)}}
$$

where $\mu(z, t)$ is the complex dilatation of $f(z, t)$. Denote by $\nu(w, t)$ the complex dilatation of the inverse mapping $f^{-1}(w, t)$; then (3) is equivalent to

$$
\bar{\partial} F(w, t)=-\frac{\partial_{t} \mu(z, t)}{\mu(z, t)} \cdot \frac{\nu(w, t)}{1-|\nu(w, t)|^{2}} \quad\left(z=f^{-1}(w, t)\right)
$$

whenever $\mu(z, t) \neq 0$.

\section{§3. Counterexample theorems}

Theorem 1. There exists a family of quasiconformal deformations $F(w, t)$ on $\bar{\Delta} \times$ $[0, T]$ such that the solution $f(z, t)$ of the system (1) and $F(w, t)$ themselves satisfy the following conditions:

(1) For $t \in\left(0, t_{1}\right]$, both $f(z, t)$ and $F(w, t)$ are extremal.

(2) For $t \in\left(t_{1}, t_{2}\right], f(z, t)$ is extremal while $F(w, t)$ is not.

(3) For $t \in\left(t_{2}, T\right]$, neither $f(z, t)$ nor $F(w, t)$ is extremal.

The example. Let $\mu$ be an extremal Beltrami differential in $\Delta$ which has a degenerating Hamilton sequence and satisfies $|\mu(z)|=\|\mu\|_{\infty}=k^{2}$ almost everywhere. Let $G \subset \Delta$ be a compact positive-measure subset. Define

$$
\mu(z, t)=t^{2} \chi_{G}(z) \mu(z)+t \chi_{\Delta-G}(z) \mu(z)
$$

for $t \in[0, T]$, where $\chi$ denotes the characteristic function of a set, while $1<T<k^{-1}$ is a fixed number.

Let $f(z, t)$ be the quasiconformal mapping of $\Delta$ onto itself with complex dilatation $\mu(z, t)$ and $f(0, t)=f(1, t)-1=0$. Noting that $\mu(z, t)$ satisfies the Hamilton-Krushkal condition iff $t \in[0,1]$, by Proposition $1, f(z, t)$ is extremal iff $t \in[0,1]$.

Define

$$
F(w, t)=\partial_{t} f \circ f^{-1}(w, t) .
$$

Then $f(z, t)$ automatically satisfy the equation (1). Now we investigate the extremality of $F(w, t)$.

By the relation (3), we get

$$
|\bar{\partial} F(f(z, t), t)|=\frac{\left|\partial_{t} \mu(z, t)\right|}{1-|\mu(z, t)|^{2}}= \begin{cases}\frac{k^{2}}{1-t^{2} k^{4}}, & z \in \Delta-G, \\ \frac{2 t k^{2}}{1-t^{4} k^{4}}, & z \in G .\end{cases}
$$


Thus, if

$$
\frac{2 t k^{2}}{1-t^{4} k^{4}}>\frac{k^{2}}{1-t^{2} k^{4}},
$$

$\bar{\partial} F(w, t)$ cannot satisfy the Hamilton-Krushkal condition, and consequently $F(w, t)$ cannot be extremal.

A direct computation will show that there exists a unique number $t_{0} \in(0,1)$ such that

$$
\frac{2 t k^{2}}{1-t^{4} k^{4}} \leq \frac{k^{2}}{1-t^{2} k^{4}} \quad \text { as } t \in\left(0, t_{0}\right]
$$

while

$$
\frac{2 t k^{2}}{1-t^{4} k^{4}}>\frac{k^{2}}{1-t^{2} k^{4}} \quad \text { as } t \in\left(t_{0}, T\right] .
$$

On the other hand, by the relation (4), we get

$$
\bar{\partial} F(w, t)= \begin{cases}-\frac{2}{t} \cdot \frac{\nu(w, t)}{1-t^{4} k^{4}}, & w \in f(\cdot, t)(G), \\ -\frac{1}{t} \cdot \frac{\nu(w, t)}{1-t^{2} k^{4}}, & w \in \Delta-f(\cdot, t)(G) .\end{cases}
$$

Ler $t \in\left(0, t_{0}\right]$. Then $f(z, t)$ is extremal and so is $f^{-1}(w, t)$. By Proposition 1 , $\nu(w, t)$ satisfies the Hamilton-Krushkal condition. Now the relations (5) and (7) imply that $\bar{\partial} F(w, t)$ also satisfies the Hamilton-Krushkal condition. Consequently, $F(w, t)$ is extremal by Proposition 1 again.

Setting $t_{1}=t_{0}, t_{2}=1$, we conclude that $F(w, t)$ satisfy all the conditions in Theorem 1. This completes the proof of Theorem 1.

By considering

$$
\mu(z, t)=t^{2} \chi_{\Delta-G}(z) \mu(z)+t \chi_{G}(z) \mu(z)
$$

in the above example, we get

Theorem 2. There exists a family of quasiconformal deformations $F(w, t)$ on $\bar{\Delta} \times$ $[0, T]$ such that the solution $f(z, t)$ of the system (1) and $F(w, t)$ themselves satisfy the following conditions:

(1) For $t \in\left(0, t_{1}\right)$, neither $f(z, t)$ nor $F(w, t)$ is extremal.

(2) For $t \in\left[t_{1}, t_{2}\right), f(z, t)$ is not extremal while $F(w, t)$ is.

(3) For $t \in\left[t_{2}, T\right]$, both $f(z, t)$ and $F(w, t)$ are extremal.

\section{$\S 4$. A SUfFiCiEnt CONDition}

Theorem 3. Let $f(z, t)$ be the solution of the system (1). If $\mu(z, t)$, the complex dilatation of $f(z, t)$, has the form $\mu(z, t)=k(t) \mu(z)$ for some differentiable function $k(t)$ with $k(0)=0, k^{\prime}(t)>0$. Then, for each fixed $t>0, f(z, t)$ is extremal iff $F(w, t)$ is extremal.

Proof. By the relation (4),

$$
\bar{\partial} F(w, t)=-\frac{k^{\prime}(t)}{k(t)} \cdot \frac{\nu(w, t)}{1-|\nu(w, t)|^{2}} .
$$

Therefore, $f(z, t)$ is extremal $\Leftrightarrow f^{-1}(w, t)$ is extremal $\Leftrightarrow \nu(w, t)$ satisfies the Hamilton-Krushkal condition (by Proposition 1) $\Leftrightarrow \nu(w, t) /\left(1-|\nu(w, t)|^{2}\right)$ satisfies the Hamilton-Krushkal condition (by Proposition 2$) \Leftrightarrow \bar{\partial} F(w, t)$ satisfies the 
Hamilton-Krushkal condition (by relation $(8)) \Leftrightarrow F(w, t)$ is extremal (by Proposition 1).

Remark. From the proof of Theorem 1, we find that the condition in Theorem 3 is not necessary.

\section{ACKNOWLEDGEMENT}

The author would like to thank the referee for valuable suggestions.

\section{REFERENCES}

1. L. V. Ahlfors, Quasiconformal deformations and mapings in $R^{n}$, J. d'Analyse Math. 30 (1976), 74-97. MR 58:11384

2. A. Beurling \& L. V. Ahlfors, The boundary correspondence under quasiconformal mappings, Acta. Math. 96 (1956), 125-142. MR 19:258c

3. F. P. Gardiner \& D. P. Sullivan, Symmetric structures on a closed curve, Amer. J. Math. 114 (1992), 683-736. MR 95h:30020

4. R. S. Hamilton, Extremal quasiconformal mappings with prescribed boundary values, Tran. Amer. Math. Soc. 138 (1969), 399-406. MR 39:7093

5. S. L. Krushkal, Extremal quasiconformal mappings, Siberian Math. J. 10 (1969), 411-418.

6. E. Reich, A quasiconformal extension using the parametric representation, J. d'Analyse Math. 54 (1990), 246-258. MR 91c:30035

7. E. Reich \& J. X. Chen, Extensions with bounded $\bar{\partial}$-derivative, Ann. Acad. Sci. Fenn. A I Math. 16 (1991), 377-389. MR 93b:30018

8. E. Reich \& K. Strebel, Extremal quasiconformal mappings with prescribed boundary values, Contributions to Analysis, A collection of papers dedicated to Lipman Bers. Academic Press, New York (1974), 375-391. MR 50:13511

9. A. Zygmund, Smooth functions, Duke Math. J. 12 (1945), 47-76. MR 7:60b

Department of Mathematics, Suzhou University, Suzhou 215006, People's Republic OF CHINA

E-mail address: ylshen@suda.edu.cn 